\title{
On the determination of the light curve parameters of detached active binaries
}

\section{The prototype RS Canum Venaticorum}

\author{
M. Rodonò ${ }^{1,2}$, A. F. Lanza ${ }^{2}$, and U. Becciani² \\ 1 Dipartimento di Fisica e Astronomia dell'Università degli Studi di Catania, Italy \\ 2 Osservatorio Astrofisico di Catania, Via Santa Sofia, 78, Città Universitaria, 95125 Catania, Italy \\ e-mail: mro@sunct.ct.astro.it, nla@sunct.ct.astro.it, ube@sunct.ct.astro.it
}

Received 5 January 2001 / Accepted 1 March 2001

\begin{abstract}
Photospheric cool spots are responsible for wave-like distortions observed in the wide-band light curves of close active binaries. They affect the determination of the photometric parameters in eclipsing systems making it necessary to accurately model the spot effects in order to estimate the true luminosity ratio, the fractionary radii and the inclination of the orbital plane in a consistent way. We address such a problem for the prototype active binary RS Canum Venaticorum, for which the availability of a long-term and accurate series of light curves allows us to minimize the starspot effects on the estimation of the quoted parameters. We have analysed the available light curve sequence by using a new parallel code we specifically designed for such an application by solving simultaneously for the system parameters and spot configuration. The uniqueness and stability problems related to the spot mapping are solved by means of a regularization approach, following the method described by Lanza et al. (1998). We evaluate the best set of photometric parameters and their confidence levels by using a statistical method based on the total $\chi^{2}$ criterion applied to the solution of the entire light curve sequence. The advantages offered by our approach include simplicity and generality with respect to other commonly used methods based on the covariance matrix. We discuss the quantitative effects of the starspots on the estimation of the parameters and compare our method and results with those of other authors. As an application of the derived results, we study the position of the components of RS CVn on the H-R diagram and compare them with computed evolutionary tracks. We find that the position of the primary component is compatible only by assuming a trigonometric parallax of $\sim 8.2$ mas, i.e., at the lower boundary of the confidence interval given by Hipparcos. Conversely, the effective temperature of the cooler component turns out to be $\sim 5 \%$ smaller than expected on the basis of its radius. The comparison with evolutionary tracks also yields an estimate of $\log \tau \simeq 9.41$ years for the age of the system. Moreover, we address the high precision with which the inclination of the orbital plane can be estimated in eclipsing systems, despite the presence of an intense starspot activity. This opens the interesting possibility of detecting precessional motions in young RS CVn binaries, the spin angular momenta of which are not yet aligned with that of the orbital motion.
\end{abstract}

Key words. binaries: close - binaries: eclipsing - stars: activity - starspots - stars: fundamental parameters methods: statistical

\section{Introduction}

The active close binaries belonging to the RS Canum Venaticorum class show a characteristic distortion of their optical light curves that is produced by the large area fraction of cool spots that covers their photospheres, up to $\sim 50 \%$ in some cases (Neff et al. 1995; O'Neal et al. 1996; Marino et al. 1999). In eclipsing systems, the flux modulation due to the spots compounds with the light variations due to the eclipses, making the modelling of the

Send offprint requests to: A. F. Lanza, e-mail: nla@sunct.ct.astro.it light curves a quite complicated task (cf., e.g., Rodonò et al. 1995). Several studies of sequences of light curves of RS CVn systems have been performed in recent years, with the main purpose of deriving information on the surface distribution of the spots and activity cycles. In the case of non-eclipsing systems, simple models based on two or three circular spots were successfully applied to study the evolution of the spot activity versus time (cf., e.g., Rodonò et al. 1986; Strassmeier et al. 1994; Henry et al. 1995; Oláh et al. 1997). However, in the case of eclipsing systems, those simple models appear to be less adequate, as shown by the analysis by Kang \& Wilson (1989), who succeeded in modelling the light curves of AR Lacertae 
and RS Canum Venaticorum only by assuming a variation of the temperature of the unspotted photospheres in different seasons. As discussed in detail by Rodonò et al. (1995) and Lanza et al. (1998), the need for a variation of the unspotted photosphere brightness arises from the fact that two circular spots are not sufficient to model both the change in the mean light level of the system and the distorted shape of the eclipses. As a matter of fact, the geometrical resolution is effectively increased during eclipses, because the moving limb of the occulting component scans the disk of the eclipsed component, making the light curve's profile more sensitive to smaller brightness inhomogeneities than outside eclipses. The extraction of such additional spatial information is indeed possible if a continuous distribution of the brightness over the stellar photospheres is allowed by the model, as demonstrated by Rodonò et al. (1995), Cameron \& Hilditch (1997) and Lanza et al. (1998). However, the eclipse mapping techniques, which are based on such an approach, have to face more difficult stability and uniqueness problems than the simple discrete-spot models. They may be solved by means of the regularizing techniques used by Doppler imaging methods to map rapidly rotating active stars (see Lanza et al. 1998). In such an approach, the photometric parameters (e.g., stellar relative radii, luminosity ratios, orbital inclination, limb darkening and so on) are assumed to be known and such techniques as the Maximum Entropy or Tikhonov regularization are applied to find a unique and stable spot map out of a set of potentially infinite continuous brightness distributions that are capable of fitting the wide-band light curve within a reasonable $\chi^{2}$ value.

Usually a sequence of light curves obtained in successive seasons is fitted with the same set of photometric parameters. In order to obtain a good fit for the whole sequence, the system parameters derived from preliminary light curve models usually need to be finely adjusted. Such a process is usually performed by searching the parameter space to find the set of parameters which minimizes the sums of the $\chi^{2}$ 's of the light curves in the sequence. In particular, such a procedure is used to find the unspotted luminosity ratio that is needed to model the light curve sequence and to derive information on the variation of the spot area versus time. The point is that the depth of the eclipses of an active binary is not constant. Both primary and secondary eclipse depths may change due to the variation of the area and the distribution of the spots, implying that the unspotted luminosity ratio (i.e., that presumably, but not necessarily, corresponding to the maximum light level of the system in a given light curve sequence), must be evaluated through a detailed modelling of the light curve sequence, including spot effects (cf. Rodonò et al. 1995; Lanza et al. 1998). Other parameters affected by the light curve distortions due to spots are the relative radii, which sometimes need to be slightly adjusted to further improve the fitting of the eclipse branches. In our previous approach, we first adjusted the luminosity ratio and then finely adjusted the relative radii to further improve the quality of the overall fit (Lanza et al. 1998). However, no simultaneous variation of more than one parameter at a time was allowed.

In consideration of such a limitation, it will be interesting to study the effect of the simultaneous variations of several parameters on the quality of the overal fit, in order to derive the best values for the photometric parameters and minimize the effects due to cool spots. In fact, if the system parameters were determined from a single light curve, the systematic errors induced by the spots would be maximum. However, when a sequence of light curves showing significant changes in the spot area and distribution is modelled, the systematic errors arising from the spot perturbations may certainly be reduced and even eliminated in some favourable circumstances.

In the case of the RS CVn systems, which are detached binaries with well defined and deep eclipses, the effects of spots on the evaluation of the system parameters has usually been neglected, because they are not particularly large (with the possible exception of the luminosity ratio, as already recognized by Popper 1988). Moreover, their treatment requires a long-term monitoring to obtain a suitably extended light curve sequence and a detailed modelling of the eclipse portions of the light curves.

For these reasons the importance of the spot effects on the determination of system parameters has been particularly addressed for semidetached and overcontact systems, where their perturbations may lead to significant errors in the estimation of the parameters. The problem is certainly relevant for Algols and W UMa binaries, where the spot-induced distortions can produce serious difficulty for reliable light curve modelling when combined with large proximity effects and continuous light variations (cf. Linnell 1991a,b,c; Wilson 1994; Kallrath et al. 1992; Kallrath 1993; Milone et al. 1995).

In the case of detached active binaries, the main emphasis has been on the study of the evolution of the spot pattern versus time, in an attempt to understand the properties of the spot activity itself. However, the time now seems ripe to undertake a study of the spot effects on the determination of the photometric and geometric parameters of the RS CVn systems. Some of them might show small changes in time as a consequence of magnetic activity or dynamic evolution in young binary systems. The changes of the stellar radius in response to the blocking of the photospheric flux due to starspots may be too small to be detectable on reasonable time scales, but some effect on the position of the star on the H-R diagram might be observable (cf. Spruit \& Weiss 1986 and Sect. 4). Moreover, in asynchronous systems, the rotational axes of the components may not be perpendicular to the orbital plane and give rise to a precessional motion and to a corresponding variation of the inclination of the orbital plane (Glebocki \& Stawikowski 1997, see Sect. 4).

In consideration of such reasons, we present in this paper a determination of the photometric parameters of the prototype active binary RS Canum Venaticorum, for which a suitably long and good quality light curve sequence is available (Rodonò et al. 1995). The main 
purpose is to derive better estimates for the stellar radii and luminosities for comparison with evolutionary models and to assess the degree of precision to which spot and activity perturbations allow us to derive geometric parameters. In order to perform this analysis, a large number of light curve fits is required, as discussed in the next section. Therefore, a parallel procedure was developed to perform the calculations on a massively parallel computer. The final output was analysed to establish confidence limits for the parameters from the final $\chi^{2}$ values. The implications for the physical status of the system components were also discussed.

\section{Light curve modelling and photometric parameter evaluation}

A subset of sixteen $V$-band light curves of RS Canum Venaticorum from Rodonò et al. (1995) was selected for our analysis from the complete set covering the years from 1963 to 1993. These light curves show the best covered primary and secondary eclipses, which can provide the basic information for determining the system's parameters. The spot modelling of these light curves was also discussed by Lanza et al. (1998), where details on the modelling methods and parameters can be found.

The orbital period of $\mathrm{RS} \mathrm{CVn}$ is not constant. The light curves were folded in phase using initial epochs close to the observations, such that the primary minima fall at zero phase, a procedure already adopted by Rodonò et al. (1995). Alternatively, phase shifts in the individual light curves may be allowed to account for the orbital period changes, as it was done by Kang \& Wilson (1989).

Our system model assumes that the distorted components are triaxial ellipsoids of semi-axes $a_{k}, b_{k}, c_{k}$, where $k=1,2$ indicates the two components. This assumption is adequate for well detached systems like RS CVn, but it is not appropriate for nearly contact or contact binaries, like W UMa's, for which a Roche model geometry should be adopted (see Kallrath \& Milone 1999). The distribution of the emergent brightness is computed taking into account the gravity-darkening effect and describing the limb-darkening with a linear law. In order to allow comparison with the previous treatment by Rodonò et al. (1995), we assumed a simplified atmospheric model with the same limb-darkening coefficients for the spotted and the unspotted photospheres. The reflection effect was treated assuming black-body re-irradiation with a given bolometric albedo. More realistic atmospheric models may be needed to model multiband data, but in our case the present approach is adequate since we analyse only $V$ band data.

In order to describe the distribution of the spot pattern, we subdivided the photosphere of each component into squared elements of angular side $s=9^{\circ}$ and we adjusted iteratively the specific intensity of each element to fit the observed light curve. The maximum brightness historically recorded in our light curve sequence was adopted as the unspotted magnitude of the system. This value was used to establish the correspondence between the scale of our computed flux and the observed $V$-band magnitudes.

It is important to note that the spot map that minimizes the $\chi^{2}$, i.e., the sum of the normalized squared differences between the observed and the computed fluxes for a given light curve, is not the best choice for the purpose of light curve modelling. As a matter of fact, due to the presence of some noise in the data, the minimum $\chi^{2}$ map certainly contains structures that are more likely related to noise fitting rather than to the actual spot distribution. Moreover, these maps are also unstable, i.e., they are quite sensitive to small changes in the data set. In order to overcome these limitations, we adopted the same approach as in photospheric Doppler imaging and introduced a regularizing functional in our solution procedure (Cameron 1992; Lanza et al. 1998). More precisely, the final spot map is found by minimizing an objective function which is a linear combination of the $\chi^{2}$ and a function which imposes some a priori constraints on the map in order to make it stable and unique. The Maximum Entropy and the Tikhonov regularizations (hereinafter ME and T, respectively) proved to be particularly suited for such a purpose. An introduction to such techniques can be found, e.g., in Narayan \& Nityananda (1986), and Tikhonov \& Arsenin (1979), Tikhonov \& Goncharsky (1987), respectively. Of course, also other regularization approaches may be used, such as the Boolean optimization adopted by Rodonò et al. (1995) or the schemes developed by Wild et al. (1994) and Berdyugina (1998).

In the present analysis we are not interested in a study of the spot distribution on the stellar surface, but in the effects of the spots in the derivation of the photometric parameters of the system. However, the spot solution may affect the derived values of those parameters and, therefore, it is important to adopt a stable scheme to calculate the spot map for a given set of parameters in order to allow a meaningful comparison of the $\chi^{2}$ values obtained with different sets of parameters. In this way the final solution, consisting of the best fitting parameters and the final spot map, is a continuous function of the data and parameter set, in the sense that small changes of the data or parameter values produce small solution changes.

In order to guarantee such a property of the solution, we model the spot distribution for each light curve by minimizing the objective function

$Q_{\mathrm{ME}}=\chi^{2}-\lambda_{\mathrm{ME}}\left(S_{1}+S_{2}\right)$

for the Maximum Entropy regularization and

$Q_{\mathrm{T}}=\chi^{2}+\lambda_{\mathrm{T}}\left(T_{1}+T_{2}\right)$

for the Tikhonov regularization. The definitions of the $\chi^{2}$ and of the entropy and Tikhonov functionals for the map of each of the two components, $S_{k}$ and $T_{k}(k=1,2)$, are given by Lanza et al. (1998). For the purpose of stabilizing the solution, in our search for the best set of photometric parameters we fixed the Lagrange multipliers at the values $\lambda_{\mathrm{ME}}=0.5$ and $\lambda_{\mathrm{T}}=40.0$, which, according to our experience, warrant almost always a good fit to the data and 
the stability of the solution. Moreover, these values are small enough to avoid significant deviation of the residual statistics from a normal distribution, as required to evaluate confidence intervals for the parameters (see below). For more details on the procedure of analysis and the minimization algorithm, we refer to Lanza et al. (1998).

In the present study we have allowed some photometric parameters to vary around the values adopted by Rodonò et al. (1995) by sampling the parameter space at discrete values. We concentrated on the geometrical parameters keeping fixed the gravity darkening, the limbdarkening and the ratio of the brightness between spotted and unspotted photospheres. To some extent this choice was forced by the fact that these three parameters are ill-constrained by photometric data in only one passband. Specifically, we sampled the parameter space for variations of: a) the inclination of the orbital plane with respect to the plane of the sky, $i$ b) the fractionary radii of the two components, $R_{1}$ and $R_{2}$; and c) the fractionary luminosities of the components $L_{1}$ and $L_{2}$. The values of the fixed and changed (marked with an asterisk) parameters and their variation intervals are listed in Table 1, together with the adopted variation step of each parameter $(\Delta q)$. The intervals of variation always enclose the values adopted by Rodonò et al. (1995). The brightness contrast of the spotted photosphere is defined as: $B_{\mathrm{s}}=I_{\mathrm{S}} / I_{\mathrm{u}}$, where $I_{\mathrm{s}}$ and $I_{\mathrm{u}}$ are the specific intensities of the spotted and unperturbed photosphere, respectively. Spots were allowed only on the cooler K2IV component, as indicated by the value of $B_{\mathrm{s}}=1$ assumed for the $\mathrm{F} 5 \mathrm{~V}$ star, following Rodonò et al. (1995).

The inclination was the only orbital parameter that was allowed to vary. The stellar relative radii were changed by $\pm 2.0 \%$, in steps of $0.5 \%$, but the figures of the ellipsoids representing the components were held fixed, because their variation is a second order effect for our purpose.

The fractionary luminosities were changed by $\pm 3 \%$, in step of $1 \%$, their variations being constrained by $\delta L_{1}+$ $\delta L_{2}=0$, to keep $L_{1}+L_{2}=1$ by definition. In Table 1 we give the intervals of variations of both $L_{1}$ and $L_{2}$ for easy reference, although what was actually varied was the ratio $L_{1} / L_{2}$. In our final sampling of the parameter space, we considered 7 values of the luminosity ratio, 9 values of the fractionary radius of the K2IV component, 7 values of the radius of the $\mathrm{F} 5 \mathrm{~V}$ component and 5 values of the inclination of the orbital plane. A total of 2205 combinations of the photometric parameters was thus analysed for each light curve, computing solutions with both Maximum Entropy and Tikhonov regularizations. In total, we computed 35280 light curve solutions for a full sampling of the selected parameter space with our data set consisting of a sequence of sixteen light curves. In order to accomplish such a large computational task, we developed a suitable parallel code that was runned on a CRAY T3E supercomputer. A maximum number of 256 DEC Alpha 21164 processors was available on the system. The code made use of the SHMEM parallel library of FORTRAN 90 to assign to each CPU the computation of the solution of a given light curve with a given set of photometric parameters, receiving in output and storing the $\chi^{2}$ values corresponding to the optimal values of the objective functions $Q_{\mathrm{ME}}$ and $Q_{\mathrm{T}}$, together with some information on the spot distribution, such as the total spotted area on the K2IV star.

On average, $32 \mathrm{CPUs}$ were used and the total CPU time required by our analysis was of 3475 hours with an average time of 6 min to compute the Maximum Entropy and Tikhonov solutions of a given light curve.

The overall quality of a given set of photometric parameters was quantified by means of the $\chi^{2}$ statistics defined as:

$$
C=\sum_{j=1}^{n_{l}} \sum_{i=1}^{m_{j}} \frac{\left(F_{i j}-D_{i j}\right)^{2}}{\sigma^{2}}
$$

where $n_{l}$ is the number of the light curves of which the sequence consists (in our case $n_{l}=16$ ), $m_{j}$ is the number of data points in the $j$ th light curve, $F_{i j}$ is the flux computed by our model, $D_{i j}$ is the flux observed at the phase $i$ th along the $j$ th light curve, and $\sigma$ is the standard deviation of the observed photometric points. In our analysis we fixed $\sigma=0.01 \mathrm{mag}$, according to the average standard deviation of the available photometric observations.

The best set of photometric parameters was selected as that corresponding to the absolute minimum of $C$. As a matter of fact, the parameter space was initially searched around the set of parameters adopted by Rodonò et al. (1995) to locate the minimum and then the sampling intervals were further expanded to explore the " $C$-landscape" around that minimum. This procedure was required because multiple relative minima can not be excluded in the present context (see Kallrath 1993; Milone 1994 and Kallrath \& Milone 1999 for a general discussion of this problem). Multiple minima are missed by the commonly used methods which perform an iterative search trying to minimize the value of $C$ on the basis of local information alone. On the other hand, our approach is capable of easily locating multiple relative minima, provided that their basins are sufficiently large to contain at least a few points of the sampling grid, thus allowing us to recognize them.

The probable errors of the best values of the parameters are readily obtained when the minimum of $C$ is determined by the differential correction method originally devised by Wilson \& Devinney (1971), by means of the covariance matrix (Wilson 1993; Kallrath et al. 1998). However, a more generally approach is possible that does not need the computation of the covariance matrix. It was originally devised for the estimation of the confidence limits of the model parameters used to fit X-ray spectra (Lampton et al. 1976; Avni 1976), but it can be readily applied to our problem of parameter estimation. In its more general formulation, the method neither requires that the model be linearly dependent on the parameters nor that the errors be normally distributed (Cash 1979). However, we shall apply it using the $\chi^{2}$ statistics defined by Eq. (3), which requires that the errors be normally distributed, but the model need not to be a linear one. 
Table 1. Geometrical and physical parameters adopted in modelling the RS CVn light curve sequence (see the text). The parameters denoted by an asterisk were allowed to vary within the indicated interval and step $(\Delta q)$

\section{Orbital Elements of RS CVn}

\begin{tabular}{lcc}
\hline Element & & $\Delta q$ \\
\hline Semi-major axis & 1.00 & \\
Eccentricity & 0.00 & \\
Inclination* (deg) & $85.35-85.75$ & 0.1 \\
Time of conjunction & mid primary eclipse & \\
Period (day) & $\sim 4.798$ & \\
\hline
\end{tabular}

Stellar and Model Parameters

\begin{tabular}{lccc}
\hline Stellar Parameter & $F 5 V$ & K2 IV & $\Delta q$ \\
& $q_{\min }-q_{\max }$ & $q_{\min }-q_{\max }$ & \\
\hline Fractionary radius* $R_{1}$ & $0.112896-0.116352$ & & 0.000576 \\
Fractionary radius* $R_{2}$ & & $0.244134-0.253998$ & 0.001233 \\
Ellipsoid semi-axis $a_{k} / R_{k}$ & 1.0 & 1.0 & \\
Ellipsoid semi-axis $b_{k} / R_{k}$ & 0.9974 & 0.9724 & \\
Ellipsoid semi-axis $c_{k} / R_{k}$ & 0.9957 & 0.9590 & \\
Mass $\left(M_{\odot}=1\right)$ & 1.39 & 1.44 & \\
Fractionary luminosity* $(V$ band $)$ & $0.4815-0.4955$ & $0.5045-0.5185$ & 0.0020 \\
Linear limb darkening & 0.56 & 0.74 & \\
Gravity darkening & 0.25 & 0.25 & \\
Bolometric albedo & 1.00 & 0.30 & \\
Albedo in the $V$ pass band & 1.01 & 0.28 & \\
Bolometric correction $(\mathrm{mag})$ & -0.04 & -0.30 & \\
Starspot brightness contrast $(V$ band $) B_{\mathrm{s}}$ & 1.0 & 0.153 & \\
\hline
\end{tabular}

We indicate with $C_{\min }$ the minimum value of $C$ corresponding to the best values of our parameters, i.e., the inclination, the fractionary radii and the luminosity ratio of the components.

The region of the parameter space which contains the true values of the four varied parameters with probability $1-\alpha$, is specified by the inequality (cf. Lampton et al. 1976, Sect. VI and Cash 1979, Sect. IIIc):

$C<C_{\min }\left[1+\frac{p}{N-p} F(p, N-p, \alpha)\right]$

where $p=4$ is the number of parameters that are varied, $N$ is the number of observations used to compute the $C$ statistics and $F$ is the Fisher-Snedecor distribution (cf., e.g., Wadsworth 1990). The main advantage of using Eq. (4) is that only the ratio $C / C_{\min }$ is considered and it is independent of the value of the average standard deviation of the observations $\sigma$, which is difficult to estimate with high accuracy.

It is important to note that the introduction of a regularization criterium for the light curve solution effectively smooths the "C-landscape", thus reducing the influence of the data noise on the parameter solution. Specifically, our final solution for the parameter best values and confidence intervals can be regarded as stable in the sense introduced above. Long-term changes in the spot pattern may introduce systematic errors in the parameter estimation that can be recognized only with a suitably long data sequence covering widely different spot configurations (see Sect. 3).

The statistical method outlined here will be applied to the analysis of our data set in the next section.

\section{Results}

Our sampling of the parameter space was performed by computing the $C$ statistics at discrete, evenly spaced points. Each of these points corresponds to a set of parameter values that represent its coordinates in the parameter space. However, it is more convenient to use integer coordinates to indicate the points of the parameter space, because it was sampled at even intervals. If we consider a point with parameter values $\left(q_{1}, q_{2}, q_{3}, q_{4}\right)$, where the $q_{i}$ 
are the values of the four photometric parameters, we can specify it by the set of four integer values $\left(j_{1}, j_{2}, j_{3}, j_{4}\right)$ defined as:

$j_{i}=\frac{\left(q_{i}-q_{i \min }\right)}{\Delta q_{i}}$

where $q_{i}$ is the given value of the $i$ th parameter, $q_{i \text { min }}$ is its minimum value in the sampled region and $\Delta q_{i}$ is its increment. In this way, the integer $j_{i}$ tells us how many increments $\Delta q_{i}$ have been given to the parameter $q_{i}$ with respect to its minimum value in our sampled region. The values of $q_{i \text { min }}$ and $\Delta q_{i}$ are reported in Table 1 and can be used to compute the values of the parameters corresponding to a point of integer coordinates $\left(j_{i}, i=1,4\right)$.

It is interesting to see which values of the photometric parameters are derived from the analysis of each light curve in our sequence. We have determined the set of parameters which minimize the $C$ statistics for each light curve $\left(n_{l}=1\right)$, both with Maximum Entropy and Tikhonov regularizations. The indexes $j_{i}$ of the parameters' sets are plotted versus the total spotted area in Fig. 1 for the ME solutions and in Fig. 2 for the T solutions, respectively. Different $j_{i}$ (i.e., different parameters) are indicated by different symbols. These plots show that the sets of best parameters derived from the single light curve analysis are different, although within the confidence intervals estimated with the use of Eq. (4). There seems to be no clear correlation between the values of the parameters and the total spot area for both ME and $\mathrm{T}$ solutions. It is interesting to note that the values of the total area given by the $\mathrm{ME}$ and $\mathrm{T}$ regularizations are different from those obtained with the Boolean regularization adopted by Rodonò et al. (1995), but the relative variations of the area from one epoch to another are very similar. This result was obtained adopting the same unspotted magnitude for all the light curves, i.e., that given by Rodonò et al. (1995).

We conclude that the effect of starspots on the best parameter sets determined from single light curves is likely that of increasing the random errors of the parameters and not of inducing systematic variations of the parameters themselves, at least in the form of simple correlations. It may be that the correlation between the spot area and the parameter values (in particular the luminosity ratio) is hidden or made non-linear by the properties of the regularization functions.

The best values of the photometric parameters can be determined from the solution of the whole light curve sequence. The values of the $C$ statistics normalized to its minimum value $C_{\text {min }}$ are shown in Fig. 3 for the ME solution and in Fig. 4 for the T solutions, respectively. In order to allow us an easy and quick comparison among different solutions, the values of $C / C_{\min }$ are plotted versus $D^{2}$, the square of the Euclidean distance of a point from the point corresponding to $C_{\min }$ in the $j$-space. More precisely, if we denote by $j_{i \text { min }}$ the values of the $j$ 's corresponding to the

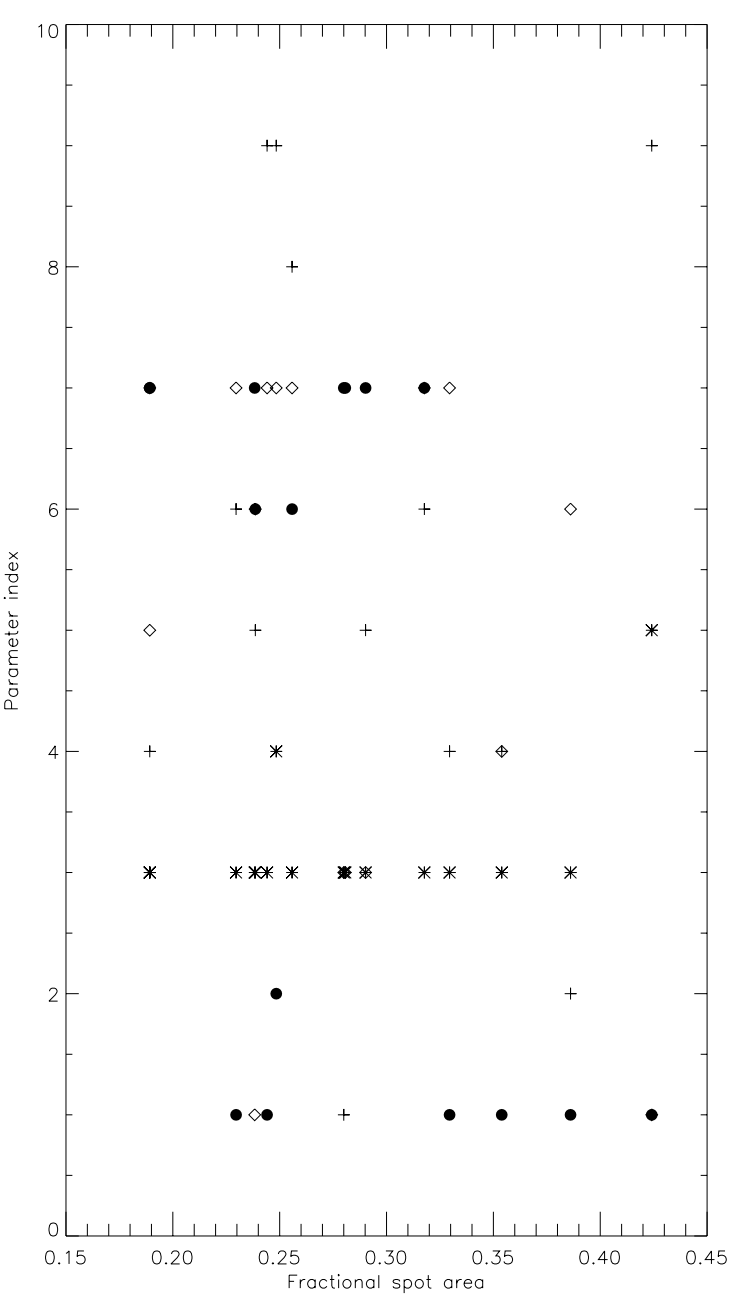

Fig. 1. The indexes $j_{i}$ indicating the best photometric parameters versus the total spot area for the Maximum Entropy solution of each light curve in our sequence. The different symbols denote the various parameters. Filled circles: luminosity ratio $L_{1} / L_{2}$; asterisks: inclination $i$; crosses: fractionary radius of the K2IV star; diamonds: fractionary radius of the F5V star. The total spotted area is expressed as a fraction of the total photospheric area

parameter set of $C_{\mathrm{min}}$, the squared distance $D^{2}$ of a point of coordinates $j_{i}$ is:

$D^{2}=\sum_{i=1}^{4}\left(j_{i}-j_{i \text { min }}\right)^{2}$.

The different symbols in Figs. 3 and 4 indicate different values of the fractionary radius of the K2IV component as specified in the caption.

The values of $C$ were normalized to the minimum value $C_{\text {min }}$, corresponding to the best set of parameters, in order to allow us to use Eq. (4) to estimate confidence intervals for the parameters. In Eq. (4), the number of varied parameters is $p=4$ and the total number of photometric points in our light curve sequence is $N=1626$. The value of $C / C_{\min }$ corresponding to $\alpha=0.01$ is indicated on the plots. The region below that limit encloses the point corresponding to the true parameter set with a probability 


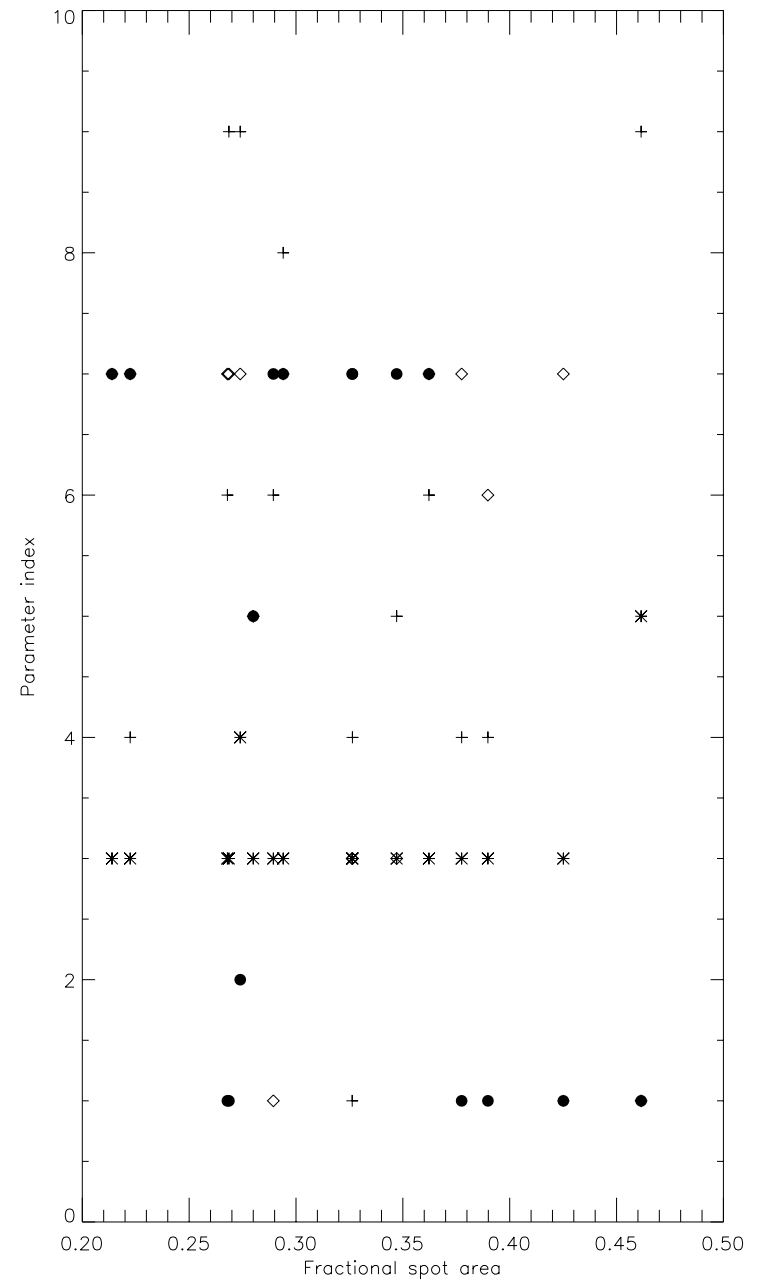

Fig. 2. The same as Fig. 1, but for the Tikhonov regularized solutions

of $99 \%$, if systematic errors do not affect the solution (see Sect. 4).

The confidence intervals for the four photometric parameters are listed in Table 2 . The same intervals were found for both $\mathrm{ME}$ and $\mathrm{T}$ regularization methods. The confidence interval for the inclination was assumed to be half of the step used in the parameter sampling, but it is likely to be even smaller because the inclination is constant for all the solutions with $C / C_{\min }<1.287$ for the ME solutions and $C / C_{\min }<1.278$ for the $\mathrm{T}$ solutions, which amply enclose the $\alpha=0.01$ confidence regions. The solutions within the confidence regions differ only for the values of the luminosity ratio and the radius of the components. More specifically, the smaller values of $C$ are characterized by a constant fractionary radius of the K2IV component with some correlation between the variation of the radius of the $\mathrm{F} 5 \mathrm{~V}$ component and its fractionary luminosity, in the sense that a larger fractionary luminosity of the F5V star tends to be associated with a larger fractionary radius. The maximum distance of the solutions from the minimum within the confidence region is $D^{2}=14$, which implies that the bottom of the well containing $C_{\min }$ in the $C$-landscape is actually a short valley along which these
Table 2. Confidence intervals of the photometric parameters (see the text)

\begin{tabular}{lc}
\hline Photometric Parameter & Confidence interval $(\alpha=0.01)$ \\
\hline Fractionary luminosity $L_{\mathrm{F} 5 \mathrm{~V}}$ & {$[0.4933,0.5162]$} \\
Fractionary luminosity $L_{\mathrm{K} 2 \mathrm{IV}}$ & {$[0.4838,0.5067]$} \\
Inclination $i$ & {$[85.5,85.6]$} \\
Fractionary radius $R_{\mathrm{F} 5 \mathrm{~V}}$ & {$[0.114048,0.116352]$} \\
Fractionary radius $R_{\mathrm{K} 2 \mathrm{IV}}$ & {$[0.247833,0.250299]$} \\
\hline
\end{tabular}

two parameters are correlated. However, the extension of the valley is well within our sampled region and we can conclude that the solution we found is stable and unique both for $\mathrm{ME}$ and $\mathrm{T}$ regularization.

The situation appears more complex for higher values of $C / C_{\min }$ because now also the variation of the fractionary radius of the $\mathrm{K} 2 \mathrm{IV}$ component seems to play a role and solutions with very similar values of $C$ can be obtained by simultaneously varying all three parameters. However, these solutions appear beyond the confidence level $\alpha=0.01$ and only for $D^{2} \geq 4$, i.e., at some distance from the absolute minimum.

If we consider even higher values of $C / C_{\min }$, the situation becomes increasingly complicated and comparable values of $C$ can be obtained even for sets of parameters with different inclinations (see Figs. 4 and 5 for the ME solutions). Some examples are shown in Fig. 5 where the values of the inclination are indicated by the numbers in brackets and the values of the fractionary radius of the K2IV component by the different plotting symbols (see the caption). It is interesting to see that the solutions tend to group into well separated subsets which are characterized by a correlation between the luminosity ratio and the fractionary radius of the $\mathrm{F} 5 \mathrm{~V}$ component. Moreover, it is really impressive to see the effects of a small variation of the inclination, because a variation of $i$ by only $\pm 0^{\circ} .2$ produces a variation of $C / C_{\min }$ by a factor of $\sim 15$.

Similar results were obtained also for the distribution of the solutions regularized with the Tikhonov functional, which are not shown here.

\section{Discussion}

The effects of starspots on the determination of the photometric parameters of the system are best clarified by the solution obtained for the individual light curves. In RS CVn systems, the proximity effects are small and also the possible effects of the short-term variability and flares are averaged out in our data set. Therefore, we can say that our parameter variation from one light curve to the other gives information primarily on the systematic errors produced by the starspots. The photometric ratio of the unspotted components may change by $\pm 3 \%$, the fractionary radii by about $2 \%$ and the inclination by $0.2 \mathrm{deg}$. Such variations do not appear to be correlated with the total spot area, probably because the position and extension 


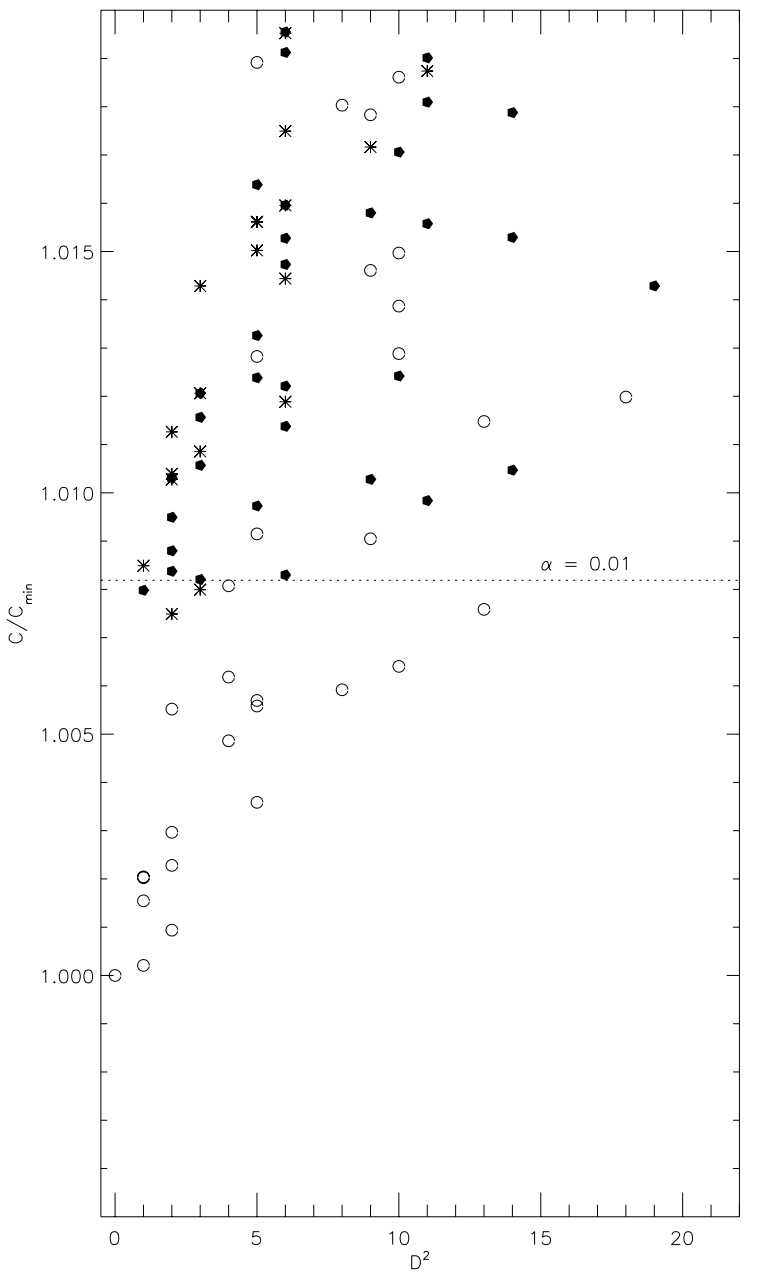

Fig. 3. The statistics $C / C_{\min }$ versus the square of the Euclidean distance $D^{2}$ from the minimum for the Maximum Entropy solutions in the neighbourhood of $C_{\min }$. The different symbols indicate different values of the index $j$ denoting the value of the variation of the fractionary radius of the K2IV component: $j=4$ (asterisks); $j=5$ (open circles); and $j=6$ (filled pentagones). The confidence level corresponding to $\alpha=0.01$ is also indicated

of the spots play some basic role in the variations. These ranges of variations are similar to those found for contact binaries by, e.g., Linnell et al. (1991a) and Kallrath et al. (1995). However, in the case of contact binaries the situation is complicated by the larger proximity effects and by other phenomena which perturb the light curve (cf. Linnel 1991b).

The simultaneous analysis of a sequence of light curves allows us to reduce the systematic errors due to spots by averaging over different surface distributions of the brightness inhomogeneities. The approach presented here is an extension of the previous analysis by Rodonò et al. (1995) and Lanza et al. (1998) to include the problem of parameter estimation. Even if the importance of accounting for starspots in parameter estimation was already recognized by Budding \& Zeilik (1987), their analysis was based on an oversimplified treatment and the modelling of only one light curve for each system, thus preventing the

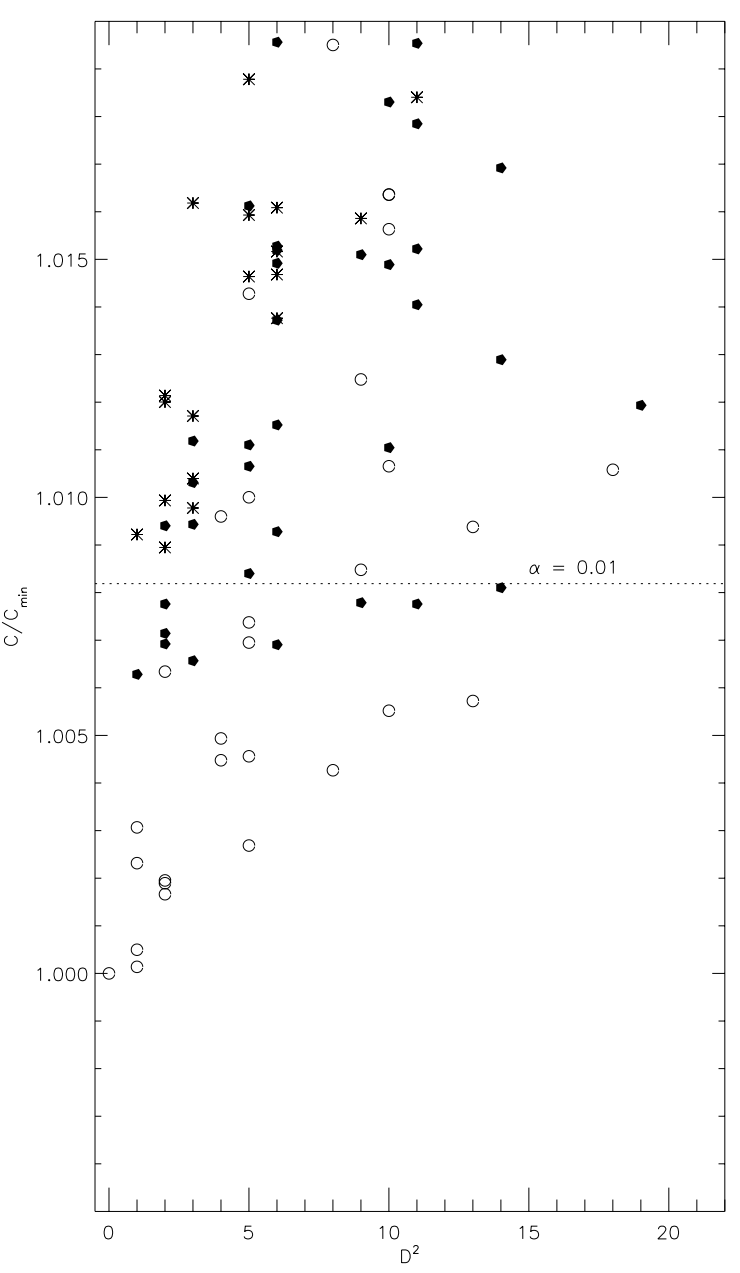

Fig. 4. The statistics $C / C_{\min }$ versus the square of the Euclidean distance $D^{2}$ from the minimum for the Tikhonov solutions in the neighbourhood of $C_{\min }$. The different symbols indicate different values of the index $j$ denoting the value of the variation of the fractionary radius of the K2IV component: $j=4$ (asterisks); $j=5$ (open circles); and $j=6$ (filled pentagones). The confidence level corresponding to $\alpha=0.01$ is also indicated

estimation of the systematic errors due to the variation of the spot pattern in time. The more sophisticated analysis by Kang \& Wilson (1989) suffered because of the limited number of light curves modelled for each system, i.e., by the short temporal interval spanned by the data, significantly shorter than typical stellar activity cycles ( $\sim 10 \mathrm{yr})$. In our present study the light curve sequence spans over an almost entire activity cycle and more than one complete migration of the photometric distortion wave on the light curve (cf. Rodonò et al. 1995), allowing us to sample the various possible starspot configurations in a more complete way. Therefore, our approach represents a further step in the direction of a consistent determination of the photometric parameters for close active binaries, as devised by Wilson (1994).

Our best solution was computed by allowing only four photometric parameters of the model to vary, i.e., the luminosity ratio of the components, their fractionary radii, and the inclination of the orbital plane. Our choice was 


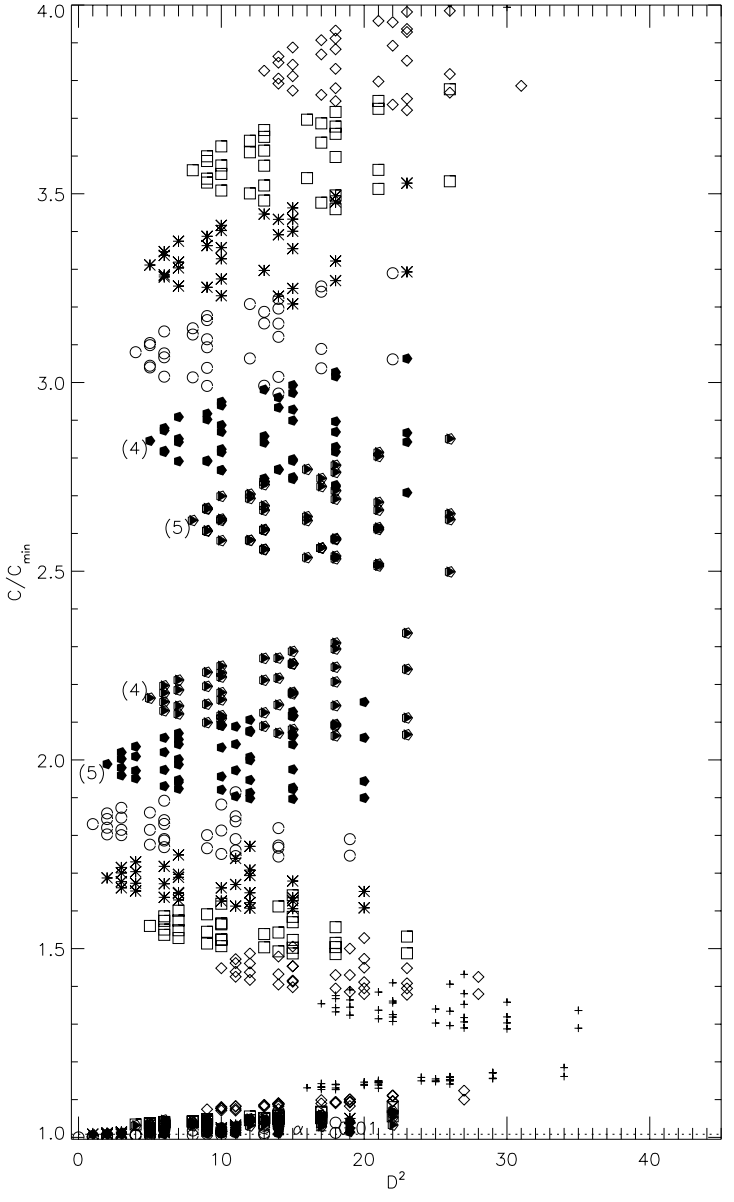

Fig. 5. The statistics $C / C_{\min }$ versus the square of the Euclidean distance $D^{2}$ from the minimum of the Maximum Entropy solutions for a larger set of $C$ and $D^{2}$ than in Fig. 3. The different symbols indicate different values of the index $j$ specifying the value of the variation of the fractionary radius of the K2IV component: $j=1$ (crosses); $j=2$ (open diamonds), $j=3$ (open squares); $j=4$ (asterisks); $j=5$ (open circles); $j=6$ (filled pentagones); $j=7$ (open pentagones); $j=8$ (filled triangles); and $j=9$ (open triangles). The confidence level corresponding to $\alpha=0.01$ is indicated by the dotted line close to the bottom of the plot. The numbers in brackets on the left of each point subset specify the values of the $j$ index of the inclination. We notice that the solutions represented by filled triangles and open pentagones are characterized by very similar values of $C / C_{\min }$ and $D^{2}$ and are therefore superimposed upon each other at the scale of the plot

justified a posteriori by the result that our solution is unique, stable and independent of the adopted regularization model. However, one may wish to know how the estimated parameters depend on the other parameters that were held fixed. Specifically, an error in the values of some of the parameters held fixed may produce systematic errors in the parameters fitted by our model. In principle, one may study the effects of a variation of one or more of the fixed parameters by extending the number of dimensions of the parameter space to be explored, but this would imply a tremendous computational effort. Moreover, the specification of parameters like the effective temperatures of the spotted and unspotted photospheres,

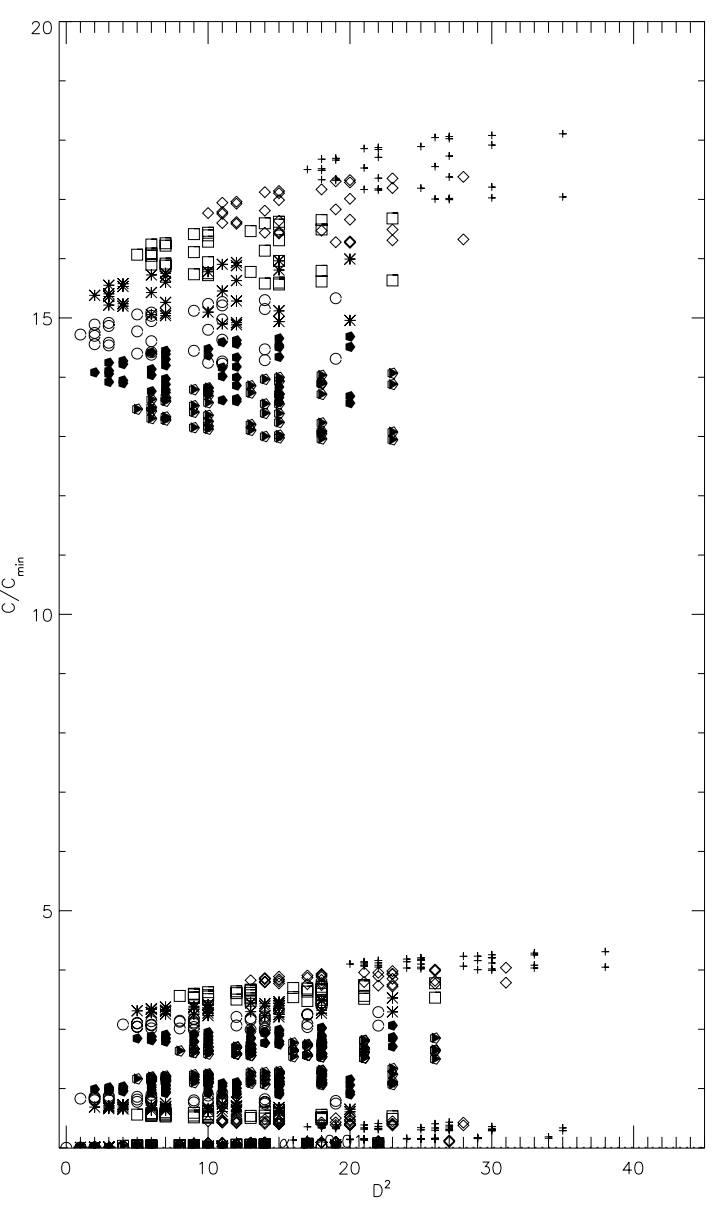

Fig. 6. The same as Fig. 5, but for a larger interval of $C$

and the limb darkening and gravity darkening coefficients, is not feasible on the basis of a data set consisting only of $V$-band light curves because they carry limited information on such parameters. If these parameters are also varied in the analysis, we expect the appearance of multiple correlations among ill-defined parameters and the loss of the uniqueness of the solution. Only if our data set had included simultaneous multi-band photometric and spectroscopic data, would that approach have been physically meaningful. Therefore, our choice of concentrating on those parameters which are directly constrained by our data set appears to be the most reasonable. We made use of the estimations by Rodonò et al. (1995) for the fixed parameters in order to extend their previous study and make the comparison easier with other studies that used the same parameter set.

An interesting point is that even if we cannot specify the true value of the photometric parameters, because of possible systematic errors, we can specify the dimensions of the confidence intervals for these parameters. This allows us to point out possible long-term variations related to stellar activity. The extension of the confidence region for the fractionary radius of the active star is $\sim 1.0 \%$; that for the inclination of the orbital plane is of 0.1 , and may possibly be made smaller; the fractionary luminosities of the two stars are uncertain by $\sim 4 \%$. Such values of the uncertainties are smaller or comparable with those estimated 
by Popper (1988) and Rodonò et al. (1995) with different methods. Therefore, variations of the parameters exceeding such amounts can be detected with a significance of $99 \%$ when a suitably long sequence of well observed light curves is available.

It is worth noticing that the extension of the confidence region changes if different weights are assigned to the photometric points. In our analysis, we gave the same statistical weight to all the observations because they are all of comparable accuracy. This choice might be questioned because photometric points within eclipses carry additional information on the photometric parameters. In the extreme case when the photometric points outside eclipses are given a null statistical weight, the total number of observations reduces to $N=522$ and the corresponding value of $C / C_{\min }$ at the $\alpha=0.01$ confidence limit is increased to: $C(\alpha=0.01) / C_{\min }=1.025$. However, even in this case, the value of the inclination is still well constrained, whereas the confidence intervals of the other parameters expand to enclose all the sampled intervals.

An application of our results on the fractionary luminosities of the components of RS CVn is the determination of the positions of the two stars on the H-R diagram by means of some assumption on the bolometric correction and the parallax derived by Hipparcos. This is interesting in view of the uncertainty about the evolutionary status of the two components of the system. Reglero et al. (1990) reported a difficulty in fitting the position of the cooler component with standard evolutionary models, whereas they found a quite good agreement for the hotter component. Barrado et al. (1994) found no difficulty in fitting the position of the stars on the H-R diagram with evolutionary models, but the effective temperatures they estimated for the components were significantly larger than those given by Reglero et al. (1990). From our determination of the unspotted fractionary luminosities in the $V$ band and the brightest magnitude of the system given by Rodonò et al. (1995), we can estimate the absolute $V$ magnitudes assuming a parallax of (9.25 11.06) mas (Perryman 1997). The bolometric magnitude can be estimated assuming a bolometric correction of $-0.66 \pm 0.1 \mathrm{mag}$ for the K2IV component and of $-0.12 \pm 0.05 \mathrm{mag}$ for the F5V component (Flower 1996).

From our solution and the available radial velocity data (Popper 1988; Eaton et al. 1993), the absolute radii and the masses of the components can be estimated with uncertainties of a few percent. The absolute bolometric magnitudes can therefore be converted into effective temperatures and the points corresponding to the components can be plotted on a radius vs. effective temperature diagram as made by Barrado et al. (1994). We show the position of the two components on the diagram in Fig. 7 together with the evolutionary tracks for different masses and chemical abundances $(Z=0.020$ and $Z=0.008)$ by Schaller et al. (1992) and Schaerer et al. (1993). The position of the F5V component can be reconciled with the evolutionary track of a mass of $M \sim 1.4 M_{\odot}$ and $Z=0.020$, if a parallax of 8.2 mas is adopted. Such a

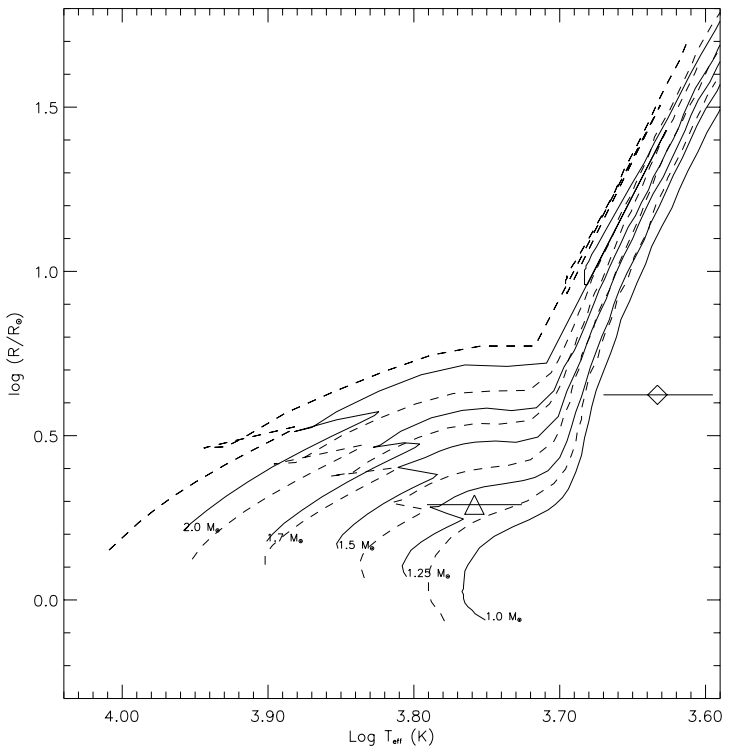

Fig. 7. Radius versus effective temperature for the components of RS CVn. The hotter F5V component is indicated by the open triangle, the cooler K2IV component by the open diamond. The uncertainties in the effective temperatures are also shown. The uncertainties in the radii are smaller than the dimension of the symbols. We also show the evolutionary tracks by Schaller et al. (1992) and Scherrer et al. (1993) for metallicities $Z=0.020$ (solid lines) and $Z=0.008$ (dashed lines). The values of the initial masses are indicated for the $Z=0.020$ models, respectively

value corresponds to a deviation of 1 standard error from the average parallax given by Hipparcos. On the other hand, the position of the cooler component cannot be reconciled with the evolutionary tracks, as already noticed by Reglero et al. (1990). Even using the latest models by Girardi et al. (2000), a discrepancy of $\sim 5 \%$ in the effective temperature still remains (see Fig. 8), unless a high metal abundance is adopted $(Z \geq 0.040)$. However, the position of the cooler component on the H-R diagram may be affected by its magnetic activity, as discussed by Spruit \& Weiss (1986). In particular, the blocking effect of the starspots may produce a reduction of the effective temperature on time intervals shorter than the thermal timescale of the convective envelope, thus explaining the position of the star on the H-R diagram.

The evolutionary tracks by Girardi et al. (2000) may also be used to estimate the age of the system through the position of the F5V component, which does not appear to be significantly active (cf. also Eaton et al. 1993). By a linear interpolation along the track corresponding to $M=$ $1.4 M_{\odot}$ and $Z=0.019$, we find a $\log \tau=9.41$ years, in agreement with the previous estimates by Reglero et al. (1990) and Barrado et al. (1994).

Another interesting application of the method of parameter estimation we adopted is the study of the precessional motion that is expected in binaries which have not yet reached the complete tidal circularization and synchronization of the spin and orbital angular momenta. Glebocki \& Stawikowski (1997) have claimed that 
several chromospherically active binaries with eccentric orbits may have a sizeable inclination of their equatorial planes with respect to their orbital planes. In the particular case of RS Canum Venaticorum, the orbit is circular and the rotation of the active star is almost perfectly synchronized with the orbital motion (Rodonò et al. 1995). Therefore, no precession of the stellar rotation axis is expected. However, it may be of some interest to estimate the observable effects and their timescales for systems which have not synchronized yet.

The moment of inertia associated with the orbital motion is typically about one hundred times larger than the moment of inertia associated with the spin of the components in RS CVn systems. Therefore, even in the case of a sizeable misalignment of the spin and orbital angular momenta, the angle between the total system angular momentum and the orbital angular momentum is of the order of $1^{\circ}$ (for a detailed discussion see Kopal 1959). The orbital angular momentum will precede around the total angular momentum with the same period of the spin precession. In the simplest case in which the other component can be approximated by a point mass, the period of the precession is given by (cf. Kopal 1959; Kovalevsky 1967):

$P_{\text {prec }}=\frac{2}{3} \frac{\left(1-e^{2}\right)}{J_{2} \cos i_{\mathrm{e}}}\left(\frac{a_{0}}{R}\right)^{2} P$

where $e$ is the eccentricity of the orbit, $a_{0}$ the semi-major axis of the orbit, $P$ the orbital period, $R$ the radius of the extended component, $J_{2}$ its adimensional gravitational quadrupole moment and $i_{\mathrm{e}}$ the inclination of its equatorial plane on the orbital plane of the binary system. In the more general case, when both components are regarded as extended bodies, other phenomena may arise, such as the nutation of their spin axes accompanied by small fluctuations of the inclination of the orbital plane with respect to the invariable plane of the system (cf. Kopal 1959, 1978). However, Eq. (7) will suffice for a rough estimation of the period of the precession of the orbital plane around the total angular momentum of the system that will produce a periodic variation of the inclination $i$ with a typical amplitude of the order of $1^{\circ}$. The gravitational quadrupole moment of an active star is mainly due to its rotation and the value of $J_{2}$ for a uniformly rotating star can be written as (cf. Kopal 1959; Kovalevsky 1967):

$J_{2}=\frac{4}{9} k_{2} \frac{\Omega^{2} R^{3}}{G M}$

where $k_{2}$ is the apsidal motion constant (Kopal 1959; Claret 1995), $\Omega$ the angular velocity of the stellar rotation, $G$ the constant of gravitation and $M$ the mass of the star. For a typical RS CVn component, we may assume $\Omega=5 \Omega_{\odot}, R=3 R_{\odot}, M=1.3 M_{\odot}, a_{0}=15 R_{\odot}$, $P \simeq 2 \pi / \Omega, e \simeq 0$ and $i_{\mathrm{e}}=45^{\circ}$, so that from Eqs. (7) and (8) the period of precession is about $10^{3} \mathrm{yr}$. This result implies that a significant variation of the inclination $i$ may indeed be detectable, if two light curve sequences well separated in time are available.

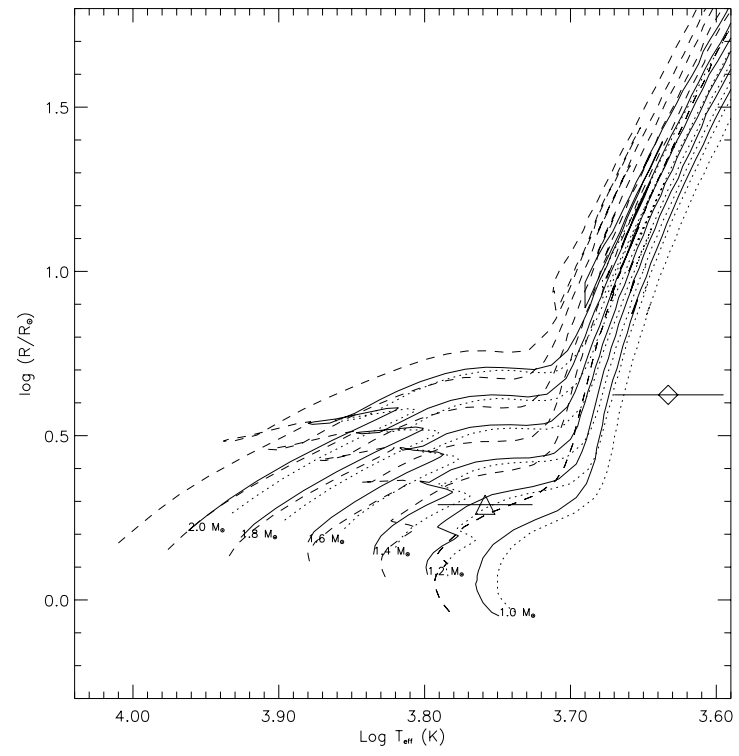

Fig. 8. Radius versus effective temperature for the components of RS CVn. The hotter F5V component is indicated by the open triangle, the cooler K2IV component by the open diamond. The uncertainties in the effective temperatures are also shown. The uncertainties in the radii are smaller than the dimension of the symbols. We also show the evolutionary tracks by Girardi et al. (2000) for metallicities $Z=0.019$ (solid lines), $Z=0.008$ (dashed lines) and $Z=0.030$ (dotted lines). The value of the initial masses are indicated for the $Z=0.019$ models, respectively

In the light of our results, we conclude that the small variation of the inclination of the orbital plane due to the precessional motion in non-synchronized close binaries can be detected with a time baseline of about one century, even for the RS CVn systems whose light curves are distorted by the presence of photospheric cool spots. The basic requirement is that the two light curve sequences consist of accurate $(\sim 0.01 \mathrm{mag})$ and well distributed observations with a total number of $N \sim 1000-1500$ points. For some systems the photometric precision may be limited by short-term changes of the spot pattern or flare activity, but a good time coverage, such as that allowed by modern automated photometric telescopes may allow us to average out such perturbations.

It is interesting to note that a close binary accompanied by a third companion may also show a periodic variation of the inclination with the period of revolution of the third body around the binary (cf. Kopal 1959). Such an effect may be detectable in a quite large fraction of cases and it may be used, together with accurate radial velocity measurements, to support or refute the third-body hypothesis often invoked to interpret the orbital period changes observed in close active binaries (cf. Lanza \& Rodonò 1999; Frasca \& Lanza 2000).

\section{Conclusion}

We have modelled a long-term sequence of light curves of $\mathrm{RS} \mathrm{CVn}$ in order to derive more reliable photometric 
parameters for the system, taking into account the light curve distortions produced by starspots. Such distortions may indeed produce systematic errors in the determinations of the parameters, as witnessed by our single light curve solutions, making questionable the parameters' values derived from the analysis of only one or a few light curves (cf. Budding \& Zeilik 1987). The analysis of several light curves obtained at different phases of the activity cycle allows us to minimize these effects.

The confidence intervals for the derived parameters were estimated using an appropriate $\chi^{2}$ statistics without the need for computing a covariance matrix.

We also discussed the positions of the two components on the H-R diagram as was determined by the derived luminosities of the two stars. The comparison with evolutionary models suggested that the effective temperature of the K2IV active component may be affected by its activity.

The high precision attainable in the determination of the orbital inclination opens the possibility of measuring the precession of the orbital plane expected in young binaries, i.e., in systems not yet synchronized. The light curve distortions induced by starspots do not appear to limit significantly such a possibility when a light curve sequence of sufficient accuracy and extension is available.

Acknowledgements. The authors wish to thank the Referee, Dr. J. Kallrath, for carefully reading their manuscript and for valuable comments. They acknowledge the CPU time on the Cray T3E supercomputer at CINECA, granted for this research project by the CNAA (Consorzio Nazionale per l'Astronomia e l'Astrofisica). They are also grateful to Vincenzo Antonuccio De Logu for several interesting discussions and to the CINECA staff for their kind assistance during the development of the present parallel code and its use.

The use of the SIMBAD and ADS databases operated by the CDS center (Strasbourg, France) is also acknowledged.

Active star research at Catania Astrophysical Observatory and the Dept. of Physics and Astronomy of Catania University is funded by MURST (Ministero della Università e della Ricerca Scientifica e Tecnologica) and Regione Siciliana, whose financial support is gratefully acknowledged.

\section{References}

Avni, Y. 1976, ApJ, 210, 642

Barrado, D., Fernández-Figueroa, M. J., Montesinos, B., \& De Castro, E. 1994, A\&A, 290, 137

Berdyugina, S. V. 1998, A\&A, 338, 97

Budding, E., \& Zeilik, M. 1987, ApJ, 319, 827

Cameron, A. C. 1992, in Surface Inhomogeneities on Late-Type Stars, ed. P. B. Byrne, \& D. J. Mullan (Springer-Verlag, Berlin), 33

Cameron, A. C., \& Hilditch, R. W. 1997, MNRAS, 287, 567

Cash, W. 1979, ApJ, 228, 939

Claret, A. 1995, A\&AS, 109, 441

Eaton, J. A., Henry, G. W., Bell, C., \& Okorogu, A. 1993, AJ, 106,1181

Flower, P. J. 1996, ApJ, 469, 355

Frasca, A., \& Lanza, A. F. 2000, A\&A, 356, 267
Girardi, L., Bressan, A., Bertelli, G., \& Chiosi, C. 2000, A\&AS, 141,371

Glebocki, R., \& Stawikowski, A. 1997, A\&A, 328, 579

Henry, G. W., Eaton, J. A., Hamer, J., \& Hall, D. S. 1995, ApJS, 97, 513

Kallrath, J. 1993, in Light Curve Modelling of Eclipsing Binary Stars, ed. Milone (Springer-Verlag, New York), 39

Kallrath, J., \& Milone, E. F. 1999, Eclipsing binary stars: modelling and analysis (Springer-Verlag, New York)

Kallrath, J., Milone, E. F., \& Stagg, C. R. 1992, ApJ, 389, 590

Kallrath, J., Milone, E. F., Terrell, D., \& Young, A. T. 1998, ApJ, 508, 308

Kang, Y. W., \& Wilson, R. E. 1989, AJ, 97, 848

Kopal, Z. 1959, Close Binary Systems (Chapman \& Hall, London)

Kopal, Z. 1978, Dynamics of Close Binary Systems (D. Reidel Publ. Co., Dordrecht, The Netherlands)

Kovalevsky, J. 1967, Introduction to Celestial Mechanics (D. Reidel Publ. Co., Dordrecht, The Netherlands)

Lampton, M., Margon, B., \& Bowyer, S. 1976, ApJ, 208, 177

Lanza, A. F., Catalano, S., Cutispoto, G., Pagano, I., \& Rodonò, M. 1998, A\&A, 332, 541

Lanza, A. F., \& Rodonò, M. 1999, A\&A, 349, 887

Linnell, A. P. 1991a, ApJ, 374, 307

Linnell, A. P. 1991b, ApJ, 379, 338

Linnell, A. P. 1991c, ApJ, 383, 330

Marino, G., Rodonò, M., Leto, G., \& Cutispoto, G. 1999, A\&A, 352,189

Milone, E. F. (ed.) 1994, Light Curve Modelling of Eclipsing Binary Stars (Springer-Verlag, New York)

Milone, E. F., Stagg, C. R., Sugars, B. A., et al. 1995, AJ, 109, 359

Narayan, R., \& Nityananda, R. 1986, ARA\&A, 24, 127

Neff, J. E., O'Neal, D., \& Saar, S. H. 1995, ApJ, 452, 879

Oláh, K., Kővári, Zs., Bartus, J., et al. 1997, A\&A, 321, 811

O'Neal, D., Saar, S. H., \& Neff, J. E. 1996, ApJ, 463, 766

Perryman, M. A. C. 1997, The Hipparcos and Tycho catalogues, ESA-SP 1200, ESA Publ. Div., Noordwijk, The Netherlands

Popper, D. M. 1988, AJ, 95, 1242

Reglero, V., Gimenez, A., \& Estela, A. 1990, A\&A, 231, 375

Rodonò, M., Cutispoto, G., Pazzani, V., et al. 1986, A\&A, 165, 135

Rodonò, M., Lanza, A. F., \& Catalano, S. 1995, A\&A, 301, 75

Schaerer, D., Meynet, G., Maeder, A., \& Schaller, G. 1993, A\&AS, 98, 523

Schaller, G., Schaerer, D., Meynet, G., \& Maeder, A. 1992, A\&AS, 96, 269

Spruit, H. C., \& Weiss, A. 1986, A\&A, 166, 167

Strassmeier, K. G., Hall, D. S., \& Henry, G. W. 1994, A\&A, 282,535

Tikhonov, A. N., \& Arsenin, V. Ya. 1979, Methods for Solving Ill-Posed Problems, Nauka, Moscow

Tikhonov, A. N., \& Goncharsky, A. V. 1987, Ill-Posed Problems in the Natural Sciences, Mir Publ., Moscow

Wadsworth, H. M. 1990, Handbook of statistical methods for engineers and scientists (McGraw-Hill Publ. Co., New York)

Wild, W. J., Rosner, R., Harmon, R., \& Drish, W. F. Jr. 1994, in Cool Stars, Stellar Systems and The Sun, Eighth Cambridge Workshop, ASP Conf. Ser., 64, ed. J.-P. Caillault, 628

Wilson, R. E. 1993, in New Frontiers in Binary Star Research, ASP Conf. Ser., 38, ed. J. C. Leung, \& I.-S. Nha, 91

Wilson, R. E. 1994, PASP, 106, 921

Wilson, R. E., \& Devinney, E. J. 1971, ApJ, 166, 605 